\title{
Preferences of epiphytic bryophytes for forest stand and substrate in North-East Latvia
}

\author{
Sanita Putna ${ }^{1} \&$ Anna Mežaka ${ }^{2}$ \\ ${ }^{1}$ Administration of Nature Protection, Rezekne, Zemnieku iela 16a, Latvia \\ E-mail: sanita.putna@inbox.lv \\ ${ }^{2}$ Research Institute for Regional Studies, Rezeknes Augstskola, \\ Atbrivosanas aleja 90, Rezekne, Latvia
}

\begin{abstract}
Distribution of epiphytic bryophyte species was studied in Woodland Key Habitats (WKH) and in managed forest stands in the North-East Latvia (district Gulbene). In total, 32 epiphytic bryophyte species were found in six WKH stands. Five endangered/threatened bryophyte species (Anomodon longifolius, Homalia trichomanoides, Jamesoniella autumnalis, Lejeunea cavifolia, Neckera pennata), that are listed in the Red Data Book of Latvia were recorded. The relation between the total and endangered/threatened epiphytic bryophyte species richness and composition with substrate factors (phorophyte species, diameter at breast height (DBH), shading, and tree bark $\mathrm{pH}$ ) was studied. The most important factors regarding epiphytic bryophyte species cover, composition and richness were found to be tree species, DBH, bark pH and forest shading. WKH indicator species richness showed significant relationship only with tree species. Tree species was one of the most important factors explaining epiphytic species distribution.
\end{abstract}

\section{INTRODUCTION}

The concept of woodland key habitat (WKH) has become an essential instrument in the biodiversity-orientated forest management in Northern Europe. This concept embraces the need to conserve the biodiversity of multi-functional forest landscapes by preserving small habitat patches that are considered to be particularly valuable (Timonen, 2010). Some WKHs in Latvia are protected. Protected forest areas in Latvia currently constitute $13.6 \%$ of all forest territory. The total forest cover in Gulbene district is 95564.9 ha; 3484.05 ha of them are protected forests and $5.4 \%$ of them are WKHs (Anonymous, 2003). The dense network of Latvian WKHs (ca. 20 per $1000 \mathrm{ha}$ ) consists of fairly large (mean area > 2 ha) WKHs (Laita et al., 2010). Approximately $50 \%$ of forests in Latvia belong to the state and other $50 \%$ are private forests. WKH inventory has been made only in state forests, but data about forest habitat quality, bryophyte and other species distribution in private owner forests is lacking (Anonymous, 2003).

It has been suggested that forest integrity might be one of conservation endpoints that integrates desirable characteristics such as biodiversity, stand structure and continuity. Epiphytic bryophytes in forest ecosystems are widely used as indicators to determine forest continuity and naturalness (Ek et al., 2002; Mežaka et al., 2010).
Phorophyte species, tree bark chemistry and structure, diameter at breast height (DBH) and exposure of tree stems are important factors that influence epiphytic bryophytes (Szövényi et al., 2004; Mežaka et al., 2008; Strazdina, 2010). The area and age of WKH have an impact on the existence of red-listed and WKH indicator species existence (Gustafsson et al., 2004). As compared to conifers, the cover of epiphytic bryophytes is much higher on deciduous tree species, such as Fraxinus excelsior, Acer platanoides, Ulmus glabra, Tilia cordata, Alnus incana and Populus tremula (Snäll et al., 2004).

Tree bark $\mathrm{pH}$ is an important factor that determines epiphyte distribution (Barkman, 1958; Weibull, 2001; Znotina, 2003). Bark pH differs among tree species and coniferous trees have a lower $\mathrm{pH}$ than deciduous trees (Smith, 1982). The composition of epiphytic species on tree stems also varies in relation to relative humidity, light intensity and bark properties (Smith, 1982; Bambe, 2002).

The objectives of the present study are: 1) to characterize and compare epiphytic bryophyte species composition in natural and managed forests in North-East Latvia, 2) identify the most important variables shaping the epiphytic bryophyte richness and composition in Gulbene district. 


\section{MATERIALS AND METHODS}

\section{Study area}

Epiphytic bryophytes were studied in different forest stands located in Gulbene district (North-East Latvia), namely Dūres mežs Nature Reserve, Kadājs Nature Reserve, Krapas gārša Nature Reserve, Pededzes lejtece Nature Reserve, Sitas un Pededzes paliene Nature Reserve and Zepu mežs Nature Reserve. Study comparing managed and WKH forest stands was carried out in Krapas gārša Nature Reserve. The sites were selected based on the WKH inventory data (Ek et al., 2002; Anonymous, 2003). The mean annual precipitation in Gulbene is $600 \mathrm{~mm}$, average temperature in January is $-7^{\circ} \mathrm{C}$ and in July $+16.4{ }^{\circ} \mathrm{C}$ (Āboltiņš, 1995).

\section{Sampling design}

The collection of bryophytes took place from 2010 to 2012 in 19 sampling plots in six forest types located randomly across the research area. In total 17 sample plots in six WKHs and two sample plots in managed forests (adjacent to four years old coppices) were studied. Two sample plots in managed mixed coniferousdeciduous forests were compared with two WKH forest sample plots of the same type. The size of sampling plot was $20 \times 20 \mathrm{~m}$. Epiphytic bryophytes were recorded up to $1.5 \mathrm{~m}$ height on 117 individual trees (with minimal DBH of $0.05 \mathrm{~m}$ ) of 15 tree species: Populus tremula (32 trees), Betula pendula (26 trees), Picea abies (18 trees), Alnus glutinosa (15 trees), Fraxinus excelsior (eight trees), Tilia cordata (six trees), Acer platanoides (five trees), Sorbus aucuparia (three trees), Sallix caprea (three trees), Frangula alnus (two trees), Ulmus laevis (two trees), Ulmus glabra (two trees), Alnus incana (two trees), Quercus robur (two trees), Pynus sylvestris (one tree). Research sites were selected based on WKH inventory data and personal observations.

Bryophyte species that were not identified in the field were collected for identification in the laboratory. For the identification of liverworts the works of Smith (1996) and Ābolina (2001) and for identification of mosses those of Ignatov \& Ignatova $(2003 ; 2004)$ were used.

Tree species, diameter at breast height, bark $\mathrm{pH}$, and height of continuous bryophyte cover on all exposures (N, W, S, and E) of tree stems were recorded. Tree bark $\mathrm{pH}$ was measured in samples that contained bryophytes on tree stem and in samples that did not contain any bryophytes, for comparison. Samples were taken at $1.3 \mathrm{~m}$ height from the stem side on which bryophytes dominated (mostly N and NW exposure). Tree bark pH was determined in the laboratory using methods developed by Kermit \& Gauslaa (2001). Bryophyte and lichen remnants were removed from tree bark to avoid their influence on $\mathrm{pH}$ value and samples of tree bark were cut. Each sample weighted approximately $0.5 \mathrm{~g}$. Bark samples were shaken in a 20-ml $1 \mathrm{M} \mathrm{KCl}$ solution for $1 \mathrm{~h}$ and $\mathrm{pH}$ was determined with a $\mathrm{pH}$-meter. All samples of one tree species were measured together (e.g. one bark pH measurement for 26 Betula pendula samples).

Shading in forest stand was defined as tree crown cover percentage in relation to a sample plot area (Eichorn et al., 2006). Four shading groups were recorded: 1) forest stand with tree crown cover over $70 \%$ (shade), 2) forest stand with tree crown cover 60-69\% (semi-shade), 3) forest stand with tree crown cover $43-59 \%$ (semi light), 4) forest stand with tree crown cover $21-42 \%$ (light).

\section{Data analysis}

Univariate Generalized linear model (GLM) with Poisson family in $\mathrm{R} 2.8 .1$ package was used to determine significant factors (tree species, DBH, exposure and tree bark $\mathrm{pH}$ ) affecting the total epiphytic species cover, total species richness and epiphytic WKH indicator species richness. Thirty-two bryophyte species and 117 trees were included in the GLM analysis.

Tree bark $\mathrm{pH}$ (154 samples) was analysed using Analysis of variance for testing treatment (with/without bryophyte cover) and tree species influence, also for testing tree species influence on bryophyte species richness. Analysis were done with $\mathrm{R}$ version 2.8.1. Differences in $\mathrm{pH}$ among tree species were not analysed due to the small number of tree replications.

Canonical Correspondence Analysis (CCA) in Canoco 4.5 for Windows was used to examine the relationship between environmental parameters (tree species, DBH, shading, exposure and tree bark $\mathrm{pH}$ ) and bryophyte species composition (Lepš \& Šmilauer 2003). Tree species and other factors were analysed separately in two CCA ordinations for decreasing noise, and their effects were compared. CCA ordination was applied to 
24 bryophyte species and 127 trees. Bryophyte and tree species with one or two occurrences were removed from the CCA analysis.

\section{RESULTS}

In total 32 epiphytic bryophyte species (25 mosses and 7 liverworts) were found on 117 trees from 15 species (Table 1). The most common bryophyte species were Hypnum cupressiforme (30 records on trees), Homalia trichomanoides (21 records), Neckera pennata (20 records) and Radula complanata (19 records). Five bryophyte species (Anomodon longifolius, Homalia trichomanoides, Jamesoniella autumnalis, Lejeunea cavifolia, Neckera pennata) listed in the Red Data Book of Latvia were found. The highest mean bryophyte richness (Fig. 1) was found on Betula

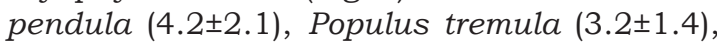
Alnus glutinosa $(2.6 \pm 0.98)$ and Picea abies $(2.4 \pm 0.84)$. No significant differences $(p>0.05)$ were found in bryophyte species richness among these tree species.

In GLM analysis (Table 2) interaction effect of tree species (seven tree species, $p<0.01$ ), bark $\mathrm{pH} \mathrm{DBH}$, and forest shading were important factors for total epiphytic bryophyte cover. Total species richness did not show any significant relation with single factors that had been studied. WKH indicator species cover depended only on tree species ( six tree species, $\mathrm{p}<0.05$ ).
Epiphytic bryophyte species composition and richness highly depended on bark $\mathrm{pH}$, exposure $(\mathrm{N}, \mathrm{NW}, \mathrm{S})$ and forest shading (Fig. 2, Fig. 3). Therefore we interpret the first CCA axis as tree bark $\mathrm{pH}$. Species on the right side of the CCA ordination (e.g. Radula complanata, Anomodon longifolius, Homalia trichomanoides, Lejeunea cavifolia, Neckera pennata) preferred higher $\mathrm{pH}$. Acidic bryophyte species on the left side of the ordination (e.g. Dicranum montanum, Ptilidium pulcherrimum, Jamesoniella autumnalis) were most often found on trees with the lowest $\mathrm{pH}$ like Betula pendula. The second CCA axis we interpreted as height on tree or epiphyte preference to substrate. For instance, Eurhynchium angustirete, Dicranum scoparium are facultative epiphytes, while Orthotrichum speciosum is an obligate epiphyte.

Bark $\mathrm{pH}$ value varied among tree species (Fig. 4). The highest mean $\mathrm{pH}$ (6.13) was found on Ulmus laevis and the lowest (3.53) on Betula pendula. Treatment (with and without bryophytes) had significant effect on bark $\mathrm{pH}$ (Table $3)$. Significant differences $(\mathrm{p}<0.05)$ in bark $\mathrm{pH}$ with moss cover were found according to Tukey's test between the tree species pairs Acer platanoides and Betula pendula, Picea abies; between Alnus glutinosa and Fraxinus excelsior, Populus tremula, Tilia cordata, B. pendula, Salix caprea, Sorbus aucupria; between B. pendula and F. excelsior, P. tremula, T. cordata, S. caprea, S.

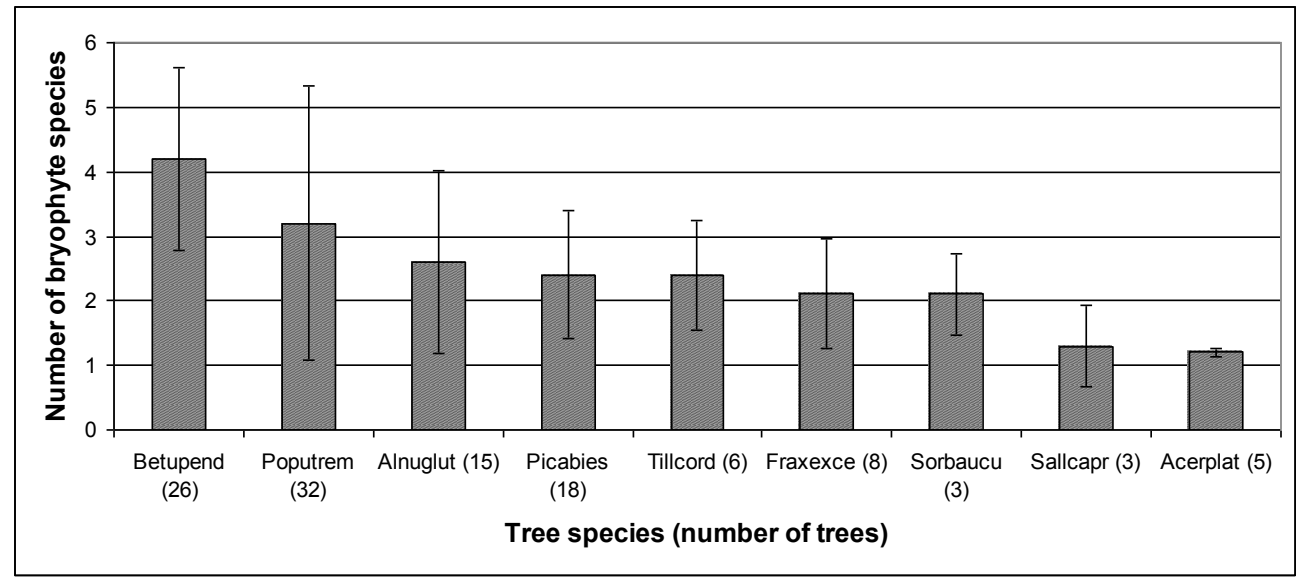

Fig. 1. Mean epiphytic bryophyte species richness on different tree species. Abbreviations: Acerplat - Acer platanoides, Alnuglut - Alnus glutinosa, Betupend - Betula pendula, Fraxexce - Fraxinus excelsior, Picabies - Picea abies, Poputrem - Populus tremula, Sallcapr - Salix caprea, Sorbaucu - Sorbus aucuparia, Tilcord - Tilia cordata. 
Table 1. Occurrence of epiphytic bryophyte species on tree species and forest type. ${ }^{*}-\mathrm{WKH}$ indicator species; liverworts in bold. Forest type abbreviations after Table 4.

\begin{tabular}{|c|c|c|c|c|c|c|c|c|c|c|c|c|c|c|c|c|}
\hline \multirow[t]{2}{*}{ Bryophyte species } & \multirow[t]{2}{*}{ Forest type } & \multicolumn{15}{|c|}{ Tree species (number of trees) } \\
\hline & & 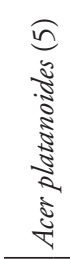 & 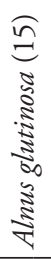 & 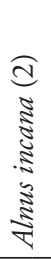 & 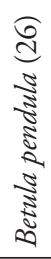 & 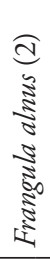 & 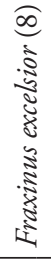 & 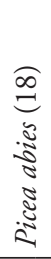 & 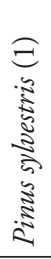 & 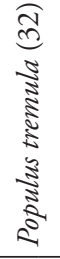 & 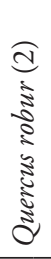 & 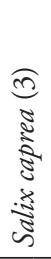 & 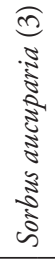 & 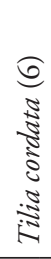 & 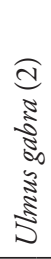 & 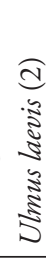 \\
\hline Amblystegium serpens & MC/DF; S/MSWF;BAWF & 1 & & & 2 & 1 & & 1 & & 1 & & 1 & & & 1 & 1 \\
\hline Anomodon longifolius* & MC/DF; DF;BAWF & & 1 & & & & 1 & & & & & & & 1 & 1 & 1 \\
\hline Brachythecium oedipodium & MC/DF; S/MSWF;BAWF & & 3 & & 1 & & & 2 & & 1 & & 1 & & & & \\
\hline Brachythecium rutabulum & MC/DF; S/MSWF;BAWF & 1 & 2 & & 1 & & & & & 1 & & & & & & \\
\hline Campylium chrysophyllum & $\mathrm{MC} / \mathrm{DF}$ & & & & & & & & & 1 & & & & & & \\
\hline Dicranum montanum & MC/DF; S/MSWF & & 1 & & 7 & & & 1 & & & & & & & & \\
\hline Dicranum scoparium & MC/DF; S/MSWF;BAWF;DF & & 3 & & 4 & & & 2 & & 4 & & & & 2 & & \\
\hline Eurhynchium angustirete & MC/DF; S/MSWF;BAWF & & 2 & & 3 & & 2 & 5 & & 4 & 1 & & & 1 & & \\
\hline Fissidens adianthoides & $\mathrm{MC} / \mathrm{DF}$ & & & & & & & & & 1 & & & & & & \\
\hline Fissidens taxifolius & MC/DF; S/MSWF;BAWF & & 2 & & & & & 1 & & 2 & & & & & & \\
\hline Homalia trichomanoides* & MC/DF; S/MSWF;DF;AF & 1 & 1 & & 1 & & 3 & & & 10 & & & & 2 & 2 & \\
\hline Homalothecium sericeum & S/MSWF & & & & 1 & & & & & & & & & & & \\
\hline Hylocomium splendens & MC/DF; S/MSWF;BAWF & 1 & 3 & & 2 & & & 6 & & 2 & & & & & & \\
\hline Hypnum cupressiforme & MC/DF; S/MSWF;DF;BAWF & 1 & 8 & 1 & 5 & & & 3 & 1 & 8 & 2 & 1 & 1 & 1 & & \\
\hline Jamesoniella autumnalis* & S/MSWF;BAWF & & & & 2 & & & & & & & & & & & \\
\hline Lejeunea cavifolia* & S/MSWF & & & & & & & & & 1 & & & 1 & & & \\
\hline Lepidozia reptans & MC/DF; S/MSWF & & 2 & & 1 & & & & & & & & & & & \\
\hline Neckera pennata* & MC/DF; S/MSWF;DF;AF & 3 & 1 & & & & 1 & & & 11 & & & 2 & 1 & 1 & \\
\hline Orthotrichum speciosum & MC/DF; S/MSWF & & & 1 & & & & & & 2 & & 1 & & & & \\
\hline Plagiochila asplenioides & MC/DF; S/MSWF;BAWF & & & & 2 & & 1 & 4 & & 6 & & & 1 & 1 & & \\
\hline Plagiochila porelloides & MC/DF; S/MSWF;BAWF & 1 & 3 & & 3 & & 1 & 1 & & 5 & & & & 1 & & \\
\hline Plagiomnium affine & MC/DF; S/MSWF & & 1 & & & & & 1 & & 2 & & & & & & \\
\hline Plagiomnium cuspidatum & MC/DF; S/MSWF;BAWF & & & & & & & 1 & & 1 & & & & & & \\
\hline Plagiothecium laetum & MC/DF; S/MSWF;BAWF & & & & 1 & & & 1 & & 4 & & & & & & \\
\hline Platygyrium repens & MC/DF; S/MSWF & & 1 & & 1 & & & & & & & & & & & \\
\hline Pleurozium schreberi & S/MSWF & & & & 2 & & & 2 & & 2 & & & & & & \\
\hline Pseudoleskeella nervosa & DF & 1 & & & & & & & & & & & & & & \\
\hline Ptilidium pulcherrimum & MC/DF; S/MSWF & & & & 4 & & & 1 & & 1 & & 1 & & 1 & & \\
\hline Pylaisia polyantha & $\mathrm{MC} / \mathrm{DF}$ & 1 & & 2 & & & & & & 1 & & & & & & \\
\hline Radula complanata & MC/DF; S/MSWF;BAWF & 1 & 2 & 1 & 3 & & 2 & & & 6 & & 1 & 1 & 1 & 1 & \\
\hline Sanionia uncinata & MC/DF; S/MSWF & & 2 & & 5 & & & & & 3 & & 1 & & & & \\
\hline Thuidium delicatulum & MC/DF; S/MSWF;BAWF & & 4 & & 2 & & & & & 2 & & & & & & \\
\hline
\end{tabular}


Table 2. Influence of variables on epiphytic bryophyte cover, GLM analysis. GLM models in bold. Betula pendula was used as a reference tree. Only significant factors $(\mathrm{p}<0.05)$ included.

\begin{tabular}{lcccc}
\hline Variables & Estimate & $\mathrm{df}$ & $\mathrm{Z}$ value & $\mathrm{p}$ \\
\hline Bryophyte cover & & & & \\
\hline Tree species+DBH+pH+shading & & \\
Intercept & 3.72 & 0.00024 & $<0.01$ \\
Tree species & & 9 & & $<0.01$ \\
Acer platanoides & 1.20 & 0.04871 & $<0.01$ \\
Alnus glutinosa & 0.55 & 0.53156 & $<0.01$ \\
Fraxinus excelsior & 0.92 & 0.00955 & $<0.01$ \\
Populus tremula & 0.64 & 0.00591 & $<0.01$ \\
Tilia cordata & 1.37 & 0.02476 & $<0.01$ \\
Ulmus glabra & 1.68 & 0.00023 & $<0.01$ \\
Ulmus laevis & -1.43 & 0.21649 & $<0.01$ \\
DBH & 0.02 & 1 & & $<0.01$ \\
pH & -0.39 & 1 & & $<0.01$ \\
Forest shading & 0.12 & 1 & & $<0.01$ \\
\hline WKH indicator species cover & & & \\
\hline Tree species & & 14 & & $<0.01$ \\
Intercept & -2.12 & 0.00024 & $<0.01$ \\
Acer platanoides & 1.61 & 0.04871 & 0.04 \\
Fraxinus excelsior & 1.83 & 0.00955 & $<0.01$ \\
Populus tremula & 1.70 & 0.00591 & $<0.01$ \\
Sorbus aucuparia & 2.12 & 0.00941 & $<0.01$ \\
Tilia cordata & 1.71 & 0.02476 & 0.02 \\
Ulmus glabra & 2.81 & 0.00023 & $<0.01$ \\
\hline & & &
\end{tabular}

Table 3. Analysis of variance regarding the influence of tree species (14) and treatment (with/ without moss cover) on bark $\mathrm{pH}$ value $(\mathrm{N}=28)$

\begin{tabular}{lrcc}
\hline Influence & F value & df & $\mathrm{p}$ \\
\hline Treatment & 146.97 & 27 & $<0.01$ \\
Tree species & 822.90 & 13 & $<0.02$ \\
Treatment ${ }^{*}$ tree species & 46.86 & & $<0.03$ \\
\hline
\end{tabular}

aucuparia; between $P$. abies and F. excelsior, $P$. tremula, T. cordata, S. caprea, S. aucuparia; between $T$. cordata and $P$. tremula. Significant differences $(\mathrm{p}<0.05)$ in bark $\mathrm{pH}$ without moss cover were found among all tree species pairs combinations.

Highest bryophyte species richness was recorded in semi-shade deciduous forests and in

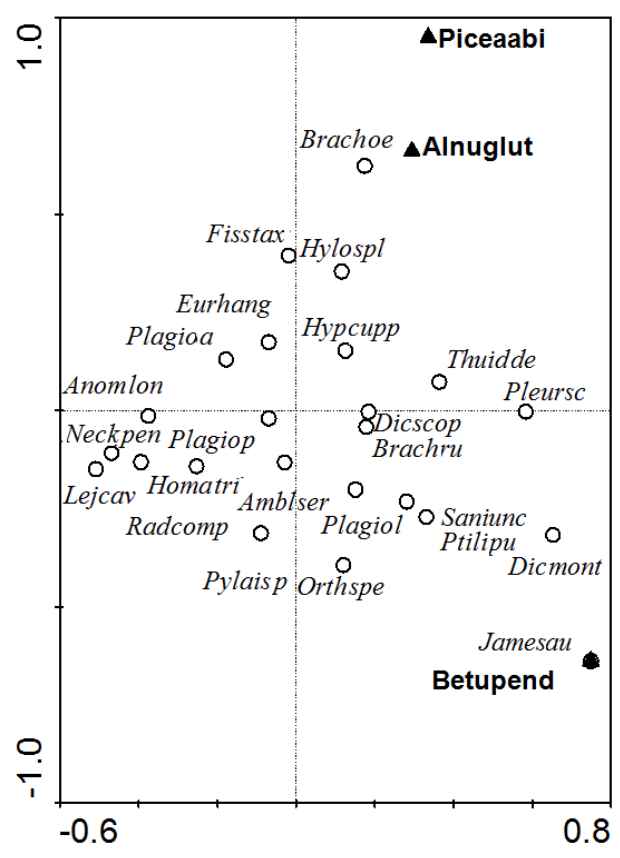

Fig. 2. CCA ordination of tree species and bryophyte species. Alnuglut - Alnus glutinosa, Betupend - Betula pendula, Piceaabi - Picea abies. Amblser - Amblystegium serpens, Anomlon-Anomodon longifolius, Brachoe - Brachythecium oedipodium, Brachru - Brachythecium rutabulum, Dicmont - Dicranum montanum, Dicscop - Dicranum scoparium, Eurhang Eurhynchium angustirete, Fisstax - Fissidens taxifolius, Homatri - Homalia trichomanoides, Hylospl - Hylocomium splendens, Hypcupp Hypnum cupressiforme, Jamesau - Jamesoniella autumnalis, Lejcav - Lejeunea cavifolia, Neckpen - Neckera pennata, Orthspe - Orthotrichum speciosum, Plagioa - Plagiochila asplenioides, Plagiop - Plagiochila porelloides, Plagiol - Plagiothecium laetum, Pleursc - Pleurozium schreberi, Ptilipu - Ptilidium pulcherrimum, Pylaisp - Pylaisia polyantha, Radcomp - Radula complanata, Sani unc - Sanionia uncinata, Thuidde - Thuidium delicatulum.

shade mixed coniferous-deciduous forests, but less bryophyte richness was found in semi-light black alder wetland forest and in light forest stands next to the coppice (Table 4, Fig. 3).

In WKH stands 19 bryophyte species were found and three of them were WKH indicator 


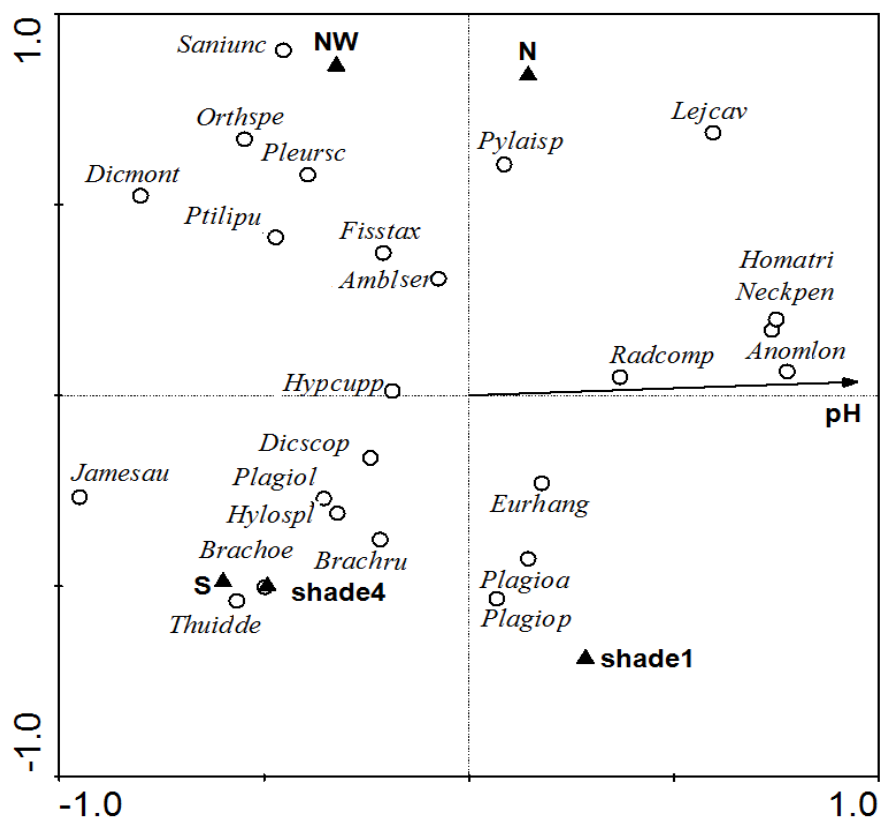

Fig. 3. CCA ordination of bryophyte species and environmental factors. Species abbreviations after Fig. 2. Environmental variables are marked as arrows and uppercase text labels. $\mathrm{pH}-\mathrm{pH}$ value of tree bark, $\mathrm{N}$ - north exposure, $\mathrm{S}$ - south exposure, NW north-west exposure, shade1 - high shading level with tree crown cover over $70 \%$, shade 4 - low shading level with tree crown cover $21-42 \%$.

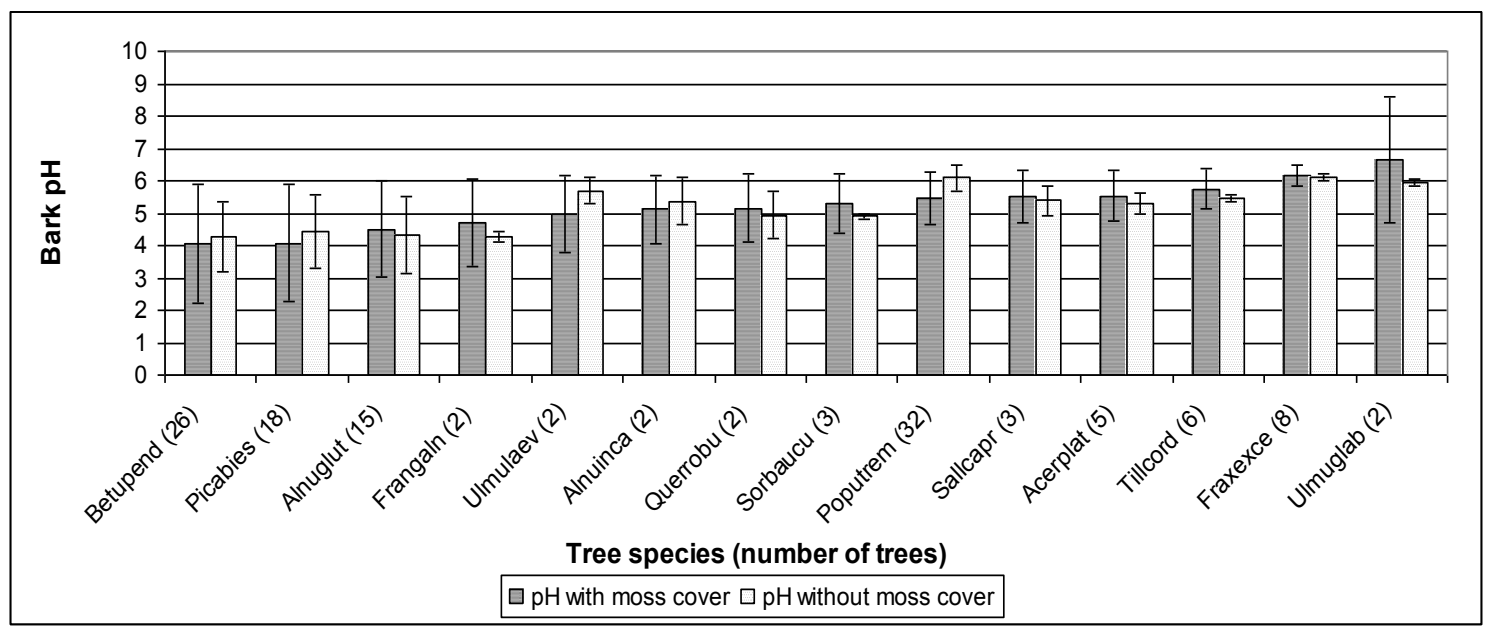

Fig. 4. Mean bark $\mathrm{pH}$ value among tree species. Abbreviations: Acerplat - Acer platanoides, Alnuglut - Alnus glutinosa, Alnuinca - Alnus incana, Betupend - Betula pendula, Fraxexce - Fraxinus excelsior, Frangaln - Frangula alnus, Picabies - Picea abies, Poputrem - Populus tremula, Querrobu - Quercus robur, Sallcapr - Salix caprea, Sorbaucu - Sorbus aucuparia, Tilcord - Tilia cordata, Ulmuglab - Ulmus glabra, Ulmulaev - Ulmus laevis. 
Table 4. Characteristics of studied territories

\begin{tabular}{|c|c|c|c|c|c|c|}
\hline Study area & Forest type/shading & $\begin{array}{l}\text { Number } \\
\text { of bryo- } \\
\text { phyte } \\
\text { species }\end{array}$ & $\begin{array}{l}\text { Mean } \\
\text { DBH } \\
(\mathrm{m})\end{array}$ & Tree species & $\begin{array}{l}\text { Forest } \\
\text { stand } \\
\text { age }\end{array}$ & $\begin{array}{r}\text { Forest } \\
\text { stand } \\
\text { area } \\
\text { (ha) } \\
\end{array}$ \\
\hline \multirow[t]{2}{*}{$\begin{array}{l}\text { Dūres mežs } \\
\text { Nature Reserve }\end{array}$} & $\begin{array}{l}\text { 1) Mixed coniferous-deciduous } \\
\text { forest (MC/DF), shade, (one } \\
\text { sample plot) }\end{array}$ & 11 & 0.36 & $\begin{array}{l}\text { Betula pendula, Picea abies, } \\
\text { Populus tremula }\end{array}$ & 76 & 2.6 \\
\hline & $\begin{array}{l}\text { 2) Aspen forest (AF), semi- } \\
\text { shade (one sample plot) }\end{array}$ & 14 & 0.57 & $\begin{array}{l}\text { Populus tremula, Sorbus } \\
\text { aucuparia }\end{array}$ & 90 & 2.4 \\
\hline \multirow[t]{2}{*}{$\begin{array}{l}\text { Kadājs Nature } \\
\text { Reserve }\end{array}$} & $\begin{array}{l}\text { 1) Spruce and mixed spruce } \\
\text { wetland forest(S/MSWF), semi } \\
\text { light, (one sample plot) }\end{array}$ & 13 & 0.26 & Betula pendula, Picea abies & 85 & 2.6 \\
\hline & $\begin{array}{l}\text { 2) Black alder wetland forest } \\
\text { (BAWF) semi light, (one sam- } \\
\text { ple plot) }\end{array}$ & 9 & 0.29 & $\begin{array}{l}\text { Alnus glutinosa, Betula pendula, } \\
\text { Picea abies }\end{array}$ & 85 & 1.7 \\
\hline \multirow[t]{3}{*}{$\begin{array}{l}\text { Krapas gārša } \\
\text { Nature Reserve }\end{array}$} & $\begin{array}{l}\text { 1) Broad-leaved forests (BLF), } \\
\text { semi-shade, (two sample plots) }\end{array}$ & 8 & 0.42 & $\begin{array}{l}\text { Acer platanoides, Alnus } \\
\text { glutinosa, Fraxinus excelsior, } \\
\text { Populus tremula, Tilia cordata. } \\
\text { Ulmus glabra }\end{array}$ & 103 & 2.1 \\
\hline & $\begin{array}{l}\text { 2) Black alder wetland forests } \\
\text { (BAWF), semi light, (two } \\
\text { sample plots) }\end{array}$ & 17 & 0.38 & $\begin{array}{l}\text { Alnus glutinosa, Betula pendula, } \\
\text { Fraxinus excelsior, Picea abies }\end{array}$ & 96 & 3.9 \\
\hline & $\begin{array}{l}\text { 3) Mixed coniferous-deciduous } \\
\text { forests (MC/DF) (five sample } \\
\text { plots in WKH-shade, and } \\
2 \text { sample plots in managed } \\
\text { stands-light) }\end{array}$ & 20 & 0.31 & $\begin{array}{l}\text { Acer platanoides, Alnus } \\
\text { glutinosa, Betula pendula, } \\
\text { Fraxinus excelsior, Picea abies, } \\
\text { Populus tremula, Quercus robur, } \\
\text { Sorbus aucuparia, Tilia cordata, } \\
\text { Ulmus glabra }\end{array}$ & 73 & 5.9 \\
\hline \multirow[t]{2}{*}{$\begin{array}{l}\text { Pededzes lejtece } \\
\text { Nature Reserve }\end{array}$} & $\begin{array}{l}\text { 1) Deciduous forests (DF), } \\
\text { semi-shade, (one sample plot) }\end{array}$ & 7 & 0.43 & $\begin{array}{l}\text { Populus tremula, Quercus robur, } \\
\text { Ulmus glabra, Fraxinus excelsior }\end{array}$ & 65 & 1.3 \\
\hline & $\begin{array}{l}\text { 2) Mixed coniferous-deciduous } \\
\text { forests (MC/DF), shade (one } \\
\text { sample plot) }\end{array}$ & 8 & 0.25 & $\begin{array}{l}\text { Alnus incana, Acer platanoides, } \\
\text { Betula pendula, Pinus sylvestris, } \\
\text { Populus tremula, Ulmus Laevis }\end{array}$ & 60 & 0.8 \\
\hline $\begin{array}{l}\text { Sitas un Ped- } \\
\text { edzes paliene } \\
\text { Nature Reserve }\end{array}$ & $\begin{array}{l}\text { Deciduous forest (DF), semi- } \\
\text { shade, (one sample plot) }\end{array}$ & 9 & 0.18 & $\begin{array}{l}\text { Alnus glutinosa, Betula pendula, } \\
\text { Frangula alnus, Salix caprea }\end{array}$ & 40 & 1.3 \\
\hline $\begin{array}{l}\text { Zepu mežs } \\
\text { Nature Reserve }\end{array}$ & $\begin{array}{l}\text { Deciduous forest (DF), semi- } \\
\text { shade, (one sample plot) }\end{array}$ & 12 & 0.34 & $\begin{array}{l}\text { Betula pendula, Populus } \\
\text { tremula, Tilia cordata }\end{array}$ & 72 & 1.8 \\
\hline
\end{tabular}

species. In managed forest 13 bryophyte species were found, among them two were WKH indicator species. In WKH forest stands bryophytes species mostly from late succession stages were found (Anomodon longifolius, Neckera pennata). In managed forest stands species from earlier succesion stages (Amblystegium serpens, Brachythecium oedipodium and B.rutabulum) and species with wide ecological amplitude (Hypnum cupressiforme, Radula complanata) were more common.

\section{DISCUSSION}

In the present study Hypnum cupressiforme, Homalia trichomanoides, Neckera pennata and Radula complanata were the most widespread bryophyte species. Radula complanata is one of the most frequent epiphytic bryophyte species in Central Sweden (Hazell et al., 1998). Homalia trichomanoides was the most common indicator species in the WKH inventory data in Latvia and Neckera pennata was also frequently found in 
the WKH inventory (Anonymous, 2003). There is, however, a need to update the information of bryophyte species in the Latvian Red Data Book with data from the recent studies, including this one. For example, Anomodon longifolius has not been previously recorded in North-East Latvia.

Significant relation was found between tree species, bark $\mathrm{pH}, \mathrm{DBH}$, shading, exposure and bryophyte species distribution. WKH indicator species richness showed significant relationship only with tree species. Tree species was one of the most important factors explaining epiphytic species distribution. The highest number of epiphytic bryophyte species was found on Populus tremula, as had been the case in several other studies in Nordic countries (Hazell et al. 1998; Snäll et al. 2004) and Latvia (Mežaka et al., 2012). Relatively high bryophyte species richness was commonly found on Betula pendula. In other studies, Betula pendula is not described as having high epiphytic species diversity (Szövényi et al., 2004). However, in our study Betula pendula was one of the most sampled tree species which could at least partly explain this trend.

Present study indicated significant bark $\mathrm{pH}$ difference with and without bryophytes. Tree bark $\mathrm{pH}$ was higher mostly under moss cover and more acidic without moss cover. On some tree species (Alnus incana, Betula pendula, Picea abies, Populus tremula, Ulmus laevis) the bark was more acidic under bryophytes. In present study tree bark $\mathrm{pH}$ is probably affected by peaty soils decreasing $\mathrm{pH}$ in black alder wetland forests, spruce and mixed spruce forest as noted also by Darell \& Cronberg (2011). The assessment of greater number of trees is necessary to get more objective results of bark $\mathrm{pH}$ value changes. Also, $\mathrm{pH}$ measurements for all individual bark samples are needed.

Managed forest stands had a lower number of epiphytic bryophyte species (13) than WKHs (19). This small difference in species richness and also presence of WKH indicator species in managed forest stand next to four years old coppice showed that disturbance did not cause immediate changes in epiphytic bryophyte species richness and community composition. However, epiphyte monitoring is necessary to see the temporal changes. In managed aspen and birch forest in Estonia the same amount of structures (dead wood, course woody debris, windfalls and windbreaks) was found as in protected forest area which corresponded to the definition of natural forests (Lõhmus et al., 2006). According to another study (Vellak \& Paal, 1999) more than twenty bryophyte species sensitive to human activity were found only in unmanaged forests. In Sweden higher bryophyte species richness was found in natural forest stands, but dominant bryophyte species and structures were the same as in managed forests (Gustafsson \& Hallingbäck, 1988).

The present study contributes to the knowledge of epiphytic bryophyte distribution and ecology in WKHs and managed forests in North-East Latvia. However, further research on different habitat management influences on epiphytic bryophyte distribution and vitality among different forest types is necessary.

\section{ACKNOWLEDGEMENTS}

We thank Inese Silamikele for all the help, advices and support in research. Thanks to Vilis Zing̣is and Jānis Naglis from Forest State Agency for information about research sites and WKH inventory data. Linda Ansone helped in the laboratory measuring bark $\mathrm{pH}$. Thanks are also given to all people, who helped in field work. We are grateful to Iveta Steinberga for help in statistical analyses and data processing. Thanks are given to Didzis Elferts for discussion about statistical methods and to Kristine Balode for revision of the English text. We are grateful to anonymous reviewers for valuable comments on the manuscript.

\section{REFERENCES}

Ābolina, A. 2001. List of bryophytes of Latvia. Latvijas Vegetäcija (in Latvian) 3: 47-87.

Āboltiňš, O. 1995. Knol of Gulbene. Encyclopedia Latvijas Daba-2, (in Latvian). Riga. 255 pp.

Anonymous, 2003. Forest inventory data of woodland key habitats in Latvia (in Latvian). 71 pp.

Bambe, B. 2002. Bryoflora of Nature Reserve "Pilskalnes Siguldiṇa”. Mežzinātne (in Latvian) 11: 111-124.

Barkman, J.J. 1958. Phytosociology and Ecology of Cryptogamic Epiphytes. Van Gorcum, Assen. $628 \mathrm{pp}$.

Darell, P. \& Cronberg N. 2011. Bryophytes in black alder swamps in south Sweden: habitat classification, environmental factors and life-strategies. Lindbergia 34: 9-29.

Eichhorn, J., Szepesi, A., Ferretti, M., Durrant, D. \& Roskams, P. 2006. Crown shading. ICP Forests Manual on Visual Assessment of Crown Condition. 167 pp. 
Ek, T., Suško, U, \& Auziñš, R. 2002. Inventory of woodland key habitats. Methodology (in Latvian). Riga. 76 pp.

Gustafsson, L. \& Hallingbäck, T. 1988. Bryophyte flora and vegetation of managed and virgin coniferous forests in South-West Sweden. Biological Conservation 44: 283-300. http: / / dx.doi. org/10.1016/0006-3207(88)90021-3

Gustafsson, L., Hylander, K. \& Jacobson, C. 2004. Uncommon bryophytes in Swedish forests-key habitats and production forests compared. Forest Ecology and Management 194: 11-22. http:/ / dx.doi.org/ 10.1016/j.foreco.2004.01.054

Hazell, P., Kellner, O., Rydin, H, \& Gustafsson, L. 1998. Presence and abundance of four epiphytic bryophytes in relation to density of aspen Populus tremula and other stand characteristics. Forest Ecology and Management 107: 147-158. http:// dx.doi.org/10.1016/S0378-1127(97)00330-7

Ignatov, M.S. \& Ignatova, E.A. 2003. Bryophyte Flora of Middle Europe in Russsia, Sphagnaceae - Hedwigiaceae, Part 1 (in Russian). Scientific Press KMK. Moscow 608 pp.

Ignatov, M.S. \& Ignatova, E.A. 2004. Bryophyte Flora of Middle Europe in Russsia, Fontinalaceae - Amblystegiaceae, Part 2 (in Russian), Scientific Press KMK. Moscow 960 pp.

Kermit, T. \& Gauslaa, Y. 2001. The vertical gradient of bark pH of twigs and microlichens in a Picea abies canopy not aff ected by acid rain. Lichenologist 35: 353-359. http://dx.doi.org/10.1006/ lich.2001.0326

Laita, A., Mönkkönen, M. \& Kotiaho, J.S. 2010. Woodland key habitats evaluated as part of a functional reserve network. Biological Conservation 143: 1212-1227. http://dx.doi.org/10.1016/j. biocon.2010.02.029

Lepš, J. \& Šmilauer, P. 2003. Multivariate analysis of ecological data using CANOCO. Cambridge. 269 pp. http://dx.doi.org/10.1017/ CBO9780511615146

Lõhmus, P., Rosenvald, R. \& Lõhmus, A. 2006. Effectiveness of solitary retention trees for conserving epiphytes: differential short-term responses of bryophytes and lichens. Canadian Journal of Forest Research 36: 1319-1330. http://dx.doi. org/10.1139/x06-032

Mežaka, A., Brūmelis, G. \& Piterāns, A. 2008. The distribution of epiphytic and lichen species in relation to phorophyte characters in Latvian natural old-growth broad leaved forests. Folia Cryptogamica Estonica 44: 89-99.
Mežaka, A., Brūmelis, G. \& Piterāns, A. 2010. Epiphytic bryophyte and lichen communities in relation to tree and forest stand variables in Populus tremula forests of south-east Latvia. Acta Biology University Daugavpils, Suppl, 2.

Mežaka, A., Brūmelis, G. \& Piterāns, A. 2012. Tree and stand scale factors affecting richness and composition of epiphytic bryophytes and lichens in deciduous woodland key habitats. Biodiversity and Conservation 21(12): 3221-3241. http:// dx.doi.org/10.1007/s 10531-012-0361-8

Smith, A.J.E. 1982. Epiphytes and epiliths, In: Bryophyte Ecology. Chapman and Hall, London, 191-227 pp.

Smith A.J.E. 1996. The Liverworts of Britain \& Ireland. Cambridge University Press, Cambridge, 362 pp.

Snäll, T., Hagström, A., Rudolphi, J. \& Rydin, H. 2004. Distribution pattern of the epiphyte Neckera pennata on three spatial scales - importance of past landscape structure, connectivity and local conditions. Ecography 27: 757-766. http://dx.doi. org/10.1111/j.0906-7590.2004.04026.x

Strazdina, L. 2010. Bryophyte community composition on an island of Lake Cieceres, Latvia: dependence on forest stand and substrate properties. Environmental and Experimental Biology 8: 49-58.

Szövényi, P., Hock, Z.S. \& Tóth, Z. 2004. Phorophyte preferences of epiphytic bryophytes in a stream valley in the Carpathian Basin. Journal of Bryology 26: 137-146. http://dx.doi. org/10.1179/037366804225021092

Timonen, J., Sitonen, J., Gustafsson, L., Kotiaho, J.S. $\&$ Stokland, J.L. 2010. Woodland key habitats in northern Europe: concepts, inventory and protection. Scandinavian Journal of Forest Research 25: 309-324. http:/ /dx.doi.org/10.1080/02827581. 2010.497160

Vellak, K. \& Paal, J. 1999. Diversity of bryophyte vegetation in some forest types in Estonia: comparison of old unmanaged and managed forests. Biodiversity and Conservation 8:1595-1620. http://dx.doi.org/10.1023/A: 1008927501623

Weibull, H. 2001. Influence of tree species on the epilithic bryophyte flora in deciduous forests of Sweden. Journal of Bryology 23: 55-56. http:// dx.doi.org/10.1179/jbr.2001.23.1.55

Znotina, V. 2003. Epiphytic bryophytes and lichens in boreal and northern temperate forests. Proceedings of the Latvian Academy of Sciences 57(1/2): $1-10$. 
84 Folia Cryptog. Estonica 